1

2

3

\title{
Effect of hybrid fiber reinforcement on the cracking process in fiber reinforced cementitious composites
}

\author{
Eduardo B. Pereira ${ }^{\mathrm{a}, *}$, Gregor Fischer ${ }^{\mathrm{b}}$, Joaquim A. O. Barros ${ }^{\mathrm{a}}$ \\ ${ }^{a}$ ISISE - University of Minho, Department of Civil Engineering, School of Engineering, \\ Azurem, 4810-058 Guimaraes, Portugal. \\ ${ }^{b}$ Technical University of Denmark (DTU), Department of Civil Engineering, Brovej, \\ building 118, DK-2800 Kgs. Lyngby, Denmark.
}

\begin{abstract}
The simultaneous use of different types of fibers as reinforcement in cementitious matrix composites is typically motivated by the underlying principle of a multi-scale nature of the cracking processes in fiber reinforced cementitious composites. It has been hypothesized that while undergoing tensile deformations in the composite, the fibers with different geometrical and mechanical properties restrain the propagation and further development of cracking at different scales from the micro- to the macro- scale. The optimized design of the fiber reinforcing systems requires the objective assessment of the contribution of each type of fiber to the overall tensile response. Possible synergistic effects resulting from particular combinations of fibers need to be clearly identified. In the present study, the evaluation of the response of different fiber reinforced cementitious composite materials is carried out by assessing directly their tensile stress-crack opening behavior. The efficiency of hybrid fiber reinforcements and the multi-scale nature of cracking
\end{abstract}

\footnotetext{
${ }^{*}$ Corresponding Author:

Email address: eduardo.pereira@civil.uminho.pt (Eduardo B. Pereira)
} 
processes are discussed based on the experimental results obtained, as well as the micro-mechanisms underlying the contribution of different fibers to bridge cracks resulting from tensile loading.

Keywords: Hybrid, Fiber Reinforcement, Tensile Properties, Cementitious Composite, Material Design, Cracking Process. 3 is governed by its susceptibility to cracking due to its quasi-brittle nature.

4 Damage tolerant concretes have been investigated since the appearance of 5 the first structural applications of fiber reinforced concretes. Pseudo-strain 6 hardening behavior in tension has particular relevance in the development 7 of these materials. The Strain Hardening Cementitious Composites (SHCC) 8 with pseudo-strain hardening ability in tension improve the durability and 9 lead to a more efficient preservation of functional properties of structures [1]. $19 \quad[2]$. 
The recent technological development of a wide variety of fibers has been creating new opportunities to the improvement of fiber reinforced cementitious composite materials. The strategy often adopted in the design of these materials is based on the utilization of fibers of different natures in the same composite. This strategy aims at designing composites with improved tensile response by taking advantage of the combined contribution of all types of fibers to the overall tensile response of the composite. The use of fibers of different natures and with distinct geometrical and material properties in hybrid fiber reinforced cementitious composites has been reported to improve the material properties of fiber reinforced cementitious composites [4-8].

The main advantage often attributed to the utilization of hybrid fiber reinforcement in fiber reinforced cementitious composites is the ability to restrain cracking at different scales of the cracking process [3,9]. It is generally recognized that the micro-mechanics of the cementitious composites is determined by the multi-scale nature of these materials, which in turn is reflected in a multi-scale structure of the cracking processes [10]. In a simplistic perspective, it is assumed that the micro-cracks generated during cracking process are bridged by smaller fibers, while the propagation of macro-cracks is restrained by the larger fibers [11]. A visible crack can be assumed as the result of the coalescence of randomly oriented and diffusely distributed microcracks previously formed. In this context, it is believed that the design of the fiber reinforcement is optimal when the multi-scale nature of the cracking process is taken into account. Further research concerning this mechanism is required though, as the explicit evidence of the true crack restraining micromechanics in multiple fiber-type reinforced cementitious composites is not 
fully established. The relation between the hierarchized cracking process in a composite, the different material scales and the mechanics of crack restraining by the different fiber reinforcements at different scales is not clearly understood.

It is the objective of the work presented in this paper to investigate in detail how fibers of different types are affecting the bridging behavior, and if a synergy between these bridging mechanisms in the composite can be established. Therefore the strategy adopted in the assessment of the tensile performance of fiber reinforced cementitious composites needs to appraise explicitly the importance and role of the micro-mechanisms of each composite phase in the overall composite response. In particular, when multiple fiber-type reinforcements are used, the contribution of each type of fiber and the interaction between different fiber reinforcements and different matrices in the overall composite mechanics needs to be clearly identified.

The tensile performance of fiber reinforced cementitious composites is typically characterized by the fracture parameters and the load-deformation behavior derived with different standard test setups [12-14]. For conventional types of fiber reinforcement, the three point bending test and the wedge splitting test setups are the most frequently used, mainly because the experimental procedure is considered simple and allows the characterization of the composite post-cracking behavior in a replicable fashion [15-18]. Inverse analysis is often utilized to derive the tensile stress-crack opening behavior from these experimental load-deformation results. Although satisfactory correlations are typically obtained, the uniqueness and universality of the solution independent of the test setup, of the boundary conditions and 
of the generated stress fields are not fully established [19]. In addition, when SHCC materials are considered, the potential formation of an unknown number of cracks during testing compromises the explicit characterization of the tensile material constitutive behavior. The direct assessment of the tensile stress-crack opening behavior, while experimentally more demanding, may be regarded as the most effective approach to access objectively the tensile performance of SHCC. In particular, when multiple types of fibers are used as reinforcement, this procedure may also allow the clear distinction of the contribution of each type of fiber to the overall composite behavior.

The assessment of the constitutive tensile stress-crack opening behavior has clear benefits in the structural design with SHCC. The numerical modeling of the mechanical behavior of SHCC at the meso-scale level may be based on micro-mechanical parameters like the mechanical properties of the fibers and of the matrix, and the properties of the fiber-matrix interface [20, 21]. However, the explicit characterization of the material behavior in terms of the stress-crack width is important to the consistent constitutive modeling [22-24]. The material design process also becomes more efficient when the direct assessment of the tensile stress-crack opening behavior is made possible. In this study, the single crack tension test (SCTT) setup is used to directly assess the tensile stress-crack opening behavior of single fiber and multiple fiber SHCC. As shown in previous research, this procedure allows the direct assessment of the tensile stress-crack opening behavior of SHCC [25-28]. The tensile responses obtained with the SCTT were also analyzed and compared with the behavior of a single crack in multiply-cracked dogbone-shaped specimens under direct tension [28]. In this study the contribution of the single 
crack tension test to understand the fracture micro-mechanisms and their influence in the tensile performance of fiber reinforced cementitious composites is analyzed, as well as its importance to support the design process of cementitious composites reinforced with multiple types of fibers.

\section{Materials and Methods}

\subsection{Materials}

The present study is focused on investigating the influence of each type of fiber on the tensile behavior of SHCC, when single or multiple types of fibers are used as reinforcement. Therefore, a similar composition of the cementitious matrix was used in all the tested composites. The cementitious matrix was mainly composed of cement (CEM 52.5 N type I), fly ash (type $\mathrm{S})$, fine sand $(0.17 \mathrm{~mm})$, quartz powder and water, with the weight proportions presented in Table 1.

Table 1: Weight of the materials used for $1 \mathrm{dm}^{3}$ of cementitious matrix.

\begin{tabular}{lllll}
\hline Cement & Fly ash & Fine sand $(0.17 \mathrm{~mm})$ & Quartz powder & Water \\
\hline $428 \mathrm{~g}$ & $856 \mathrm{~g}$ & $150 \mathrm{~g}$ & $150 \mathrm{~g}$ & $320 \mathrm{~cm}^{3}$ \\
\hline
\end{tabular}

Fibers of three different natures were used: PVA (polyvinyl alcohol), PAN (polyacrylnitrile) and PP (polypropylene). The main geometrical and mechanical properties of these fibers are presented in Table 2 
Table 2: Main properties of the fibers used.

\begin{tabular}{lrrr}
\hline Fiber & $\begin{array}{r}\text { Tensile strength } \\
(\mathrm{MPa})\end{array}$ & $\begin{array}{r}\text { Length } \\
(\mathrm{mm})\end{array}$ & $\begin{array}{r}\text { Diameter } \\
(\mu \mathrm{m})\end{array}$ \\
\hline PVA 15 & 1600 & 8 & 40.0 \\
PP & 900 & 12 & 40.0 \\
PAN 1.5 & 826 & 6 & 12.7 \\
\hline
\end{tabular}

Considering the extensive experience available with PVA fibers in the design of SHCC (Li2003), a composite formulation containing 2\% of PVA fibers was studied. This composite represents a performance reference, when the objective of accomplishing strain hardening ability in tension is considered. As reported in a previous study [28], the composite reinforced with $2 \%$ of PVA fibers may be classified as a SHCC. For reference, a composite with $1 \%$ of PVA fiber reinforcement was also investigated. The volumetric percentages of the six composites tested are presented in Table 3.

The experimental characterization of the multiple cracking behavior of SHCC is typically carried out using the direct tension tests of dogbone-shaped specimens. The SCTT approaches the problematic of the tensile behavior of these composites from a different perspective, that is, by investigating the tensile behavior of the composite at the level of a single crack. The requirements for the attainment of a single crack with the SCTT were discussed in a previous research study [28]. The SCTT results of the $2 \%$ PVA fiber reinforced SHCC were analyzed and compared with the direct tension test results of dogbone-shaped specimens. In particular, the behavior of a single 
Table 3: Fiber reinforcement volumetric percentages of the developed fiber reinforced cementitious composites.

\begin{tabular}{lccc}
\hline \multirow{2}{*}{ Composite } & $\begin{array}{c}\text { PVA 15 } \\
\text { \% vol. }\end{array}$ & $\begin{array}{c}\text { PAN 1.5 } \\
\text { \% vol. }\end{array}$ & $\begin{array}{c}\text { PP vol. } \\
\text { 1PVA }\end{array}$ \\
\hline 2PVA & 2 & 0 & 0 \\
HybPAN & 1 & 0 & 0 \\
2PAN & 0 & 2 & 0 \\
HybPP & 1 & 0 & 1.25 \\
$2.5 P P$ & 0 & 0 & 2.5 \\
\hline
\end{tabular}

crack in the multiply-cracked dogbone shaped specimen was described during tensile testing and compared with the SCTT results of the same composite $[28]$.

The adoption of $2.5 \%$ fiber volume in the case of the PP fiber reinforced composite aimed at partly compensating the lower strength of PP fibers compared to the PVA fibers. The composite specific tensile strength obtained with a volume fraction of $2 \%$ of PVA fiber reinforcement is equivalent to $3.56 \%$ of PP fibers. However, during the mixing process the fiber dispersion was found to be unsatisfactory for PP fiber volume fractions greater than $2.5 \%$. To achieve satisfactory fiber dispersion with greater fiber volumetric percentages the matrix composition would have to be redesigned. This procedure was not considered in this study, whereas the same matrix composition in all composites was necessary to analyze the composite tensile behaviors and their direct relation with the type of fiber reinforcement used. The lower 
strength of PAN fibers would also require an increase of the PAN fiber volume fraction used, to obtain a similar composite specific tensile strength. Due to their high specific surface, PAN fibers also showed strong interaction with the fresh matrix, and satisfactory fiber dispersion was observed only for fiber volume fractions not exceeding $2 \%$.

\subsection{Compression test}

When investigating the tensile performance of conventional fiber reinforced cementitious composites, the information obtained from compression tests is useful in the sense that it is predominantly associated with the mechanical properties of the matrix exclusively. The influence of fiber reinforcement on the composite compressive strength is commonly assumed as negligible, although it contributes to increase the post-cracking energy dissipation ability [29]. The fibers used in the present study are, however, substantially smaller than the ones typically observed in conventional fiber reinforced cementitious composites.

The compactness of the solid skeleton is known to affect the composite compressive strength. In particular, at the scale of the paste material structure, the microscopic arrangement of the particles of cement, fly ash and other fine additions affects the mechanical properties of the matrix [30]. Considering that the diameters of the fibers studied are of the same order of magnitude as the fine particles composing the paste, fibers will have an effect on the compactness of the solid skeleton at the scale of the paste material structure. The chemistry of fibers and fine particles will also affect the balance of interaction forces between the fine particles in suspension composing the fresh paste. Therefore, the different diameters and chemical composition 
of the fibers studied will likely have an effect on the fresh properties and on the micro-structure of the hardened composite. The compressive strength may consequently differ in all composites, although the composition of the matrix is the same. To analyze this aspect of the mechanical behavior of the composites studied, the compressive strength was evaluated.

The experimental procedure consisted of loading the specimens at a compressive displacement rate of $0.1 \mathrm{~mm} / \mathrm{s}$. For each composite three cubes $\left(40 \times 40 \times 40 \mathrm{~mm}^{3}\right)$ were tested.

\subsection{Single Crack Tension Test (SCTT)}

The mechanical characterization of a crack during the initiation and propagation stages requires the observation of a single crack during the entire loading hardening-softening test sequence. This is often a problem, given that SHCC materials are typically designed to develop multiple cracks in tension.

The conditions necessary to obtain a single crack with the SCTT for the assessment of the mechanical tensile stress-crack opening behavior of SHCC were analyzed in a previous work [27]. The SCTT results were analyzed and compared with the behavior of a single crack in multiply-cracked dogboneshaped specimens under direct tension [28]. In the present study, the SCTT procedure was adopted to assess the tensile stress-crack opening behavior of cementitious composites reinforced with single and multiple types of fibers. The geometry of the specimen is represented in Figure 1. The length of the specimen was $120 \mathrm{~mm}$ and the free distance between the clamped edges of the specimen during testing was $70 \mathrm{~mm}$. The test setup and support condi- 
197

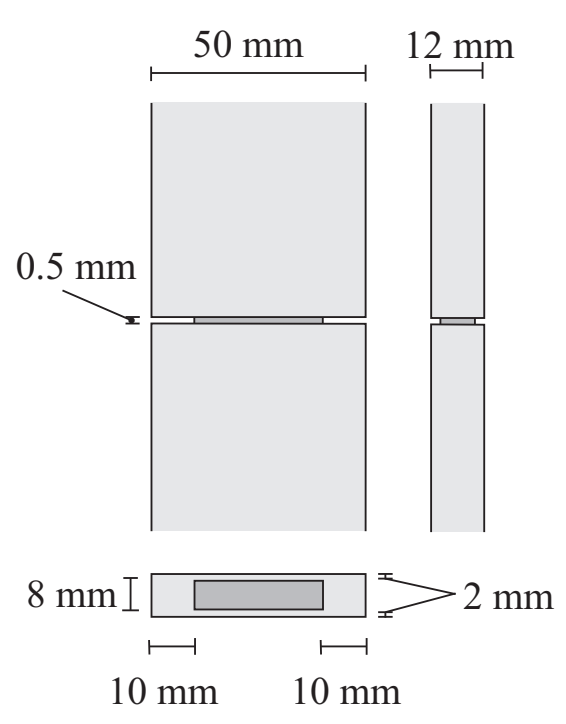

Figure 1: Geometry used in the assessment of the tensile stress-crack opening behavior.

tions are shown in Figure 2.

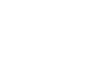

The procedure consisted of loading the specimen at a tensile displacement rate of $5 \mathrm{~m} / \mathrm{s}$. This displacement rate was transmitted to the specimen by means of two hydraulic grips, clamping the ends of the specimen (rotations and transverse displacements were restrained). During testing, the opening of the notch was measured using two clip gauges, positioned on opposite sides of the notch, as shown in Figure 2. The formation of a single crack was confirmed by inspection of the tensile stress-crack opening responses as well as visually, as shown in Figure 3(a) and Figure 3(b). 


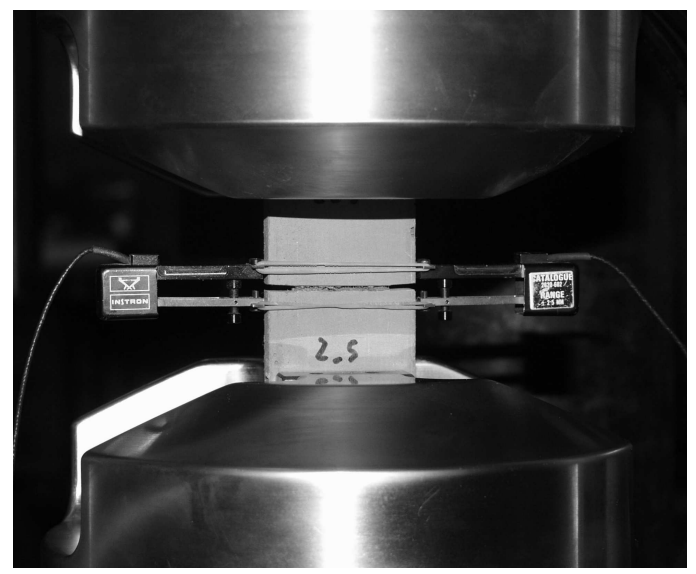

Figure 2: Tensile test setup, including supports and measuring instrumentation (clip gages).

\section{Experimental results}

For each composite, the tensile stress-crack opening behavior was derived using six specimens. The tensile stress was computed by dividing the tensile load by the net area of the notched cross-section. The crack opening was computed by averaging the notch opening recorded by the clip gauges. The opening at the opposite sides of the notch were later confirmed to be nearly identical, as a result of the imposed boundary conditions. The tensile characteristics of each composite, in the form of a tensile stress-crack opening relationship, are expected to assume the schematic shape of a multi-linear diagram as represented in Figure 4.

The overall shape of the bridging stress, $\sigma_{B}$, versus the crack opening, $w$, curve is expected to be sensitive to the simultaneous use of different types of fibers as reinforcement, causing the formation of multiple bridging stress 

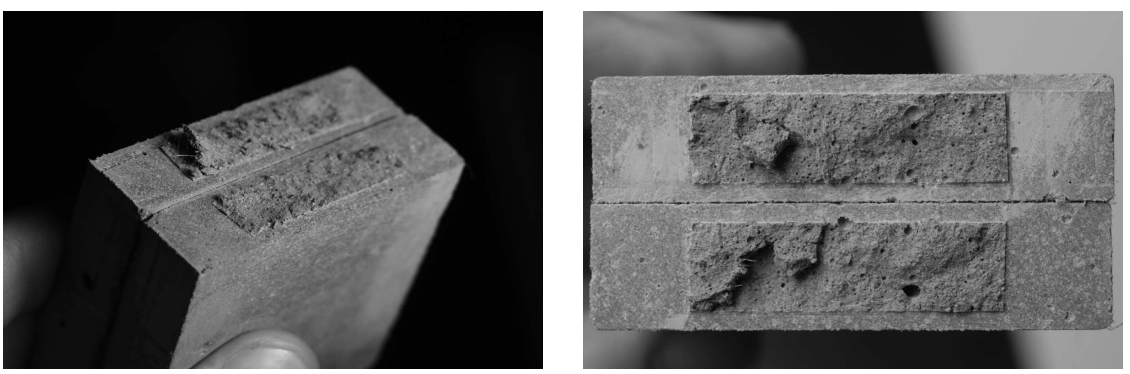

(a) Composite containing $2 \%$ of PVA (b) Composite containing 1\% PVA + fibers.

$1 \%$ PAN

Figure 3: Crack surface of specimens.

peaks. The main parameters defining the schematic of the bridging stress, $\sigma_{B}$, versus the crack opening, $w$, relationship are the first cracking stress, $\sigma_{c r}$, the initial bridging stress, $\sigma_{b, 1}$, the peak bridging stress, $\sigma_{B, 1}$, and the corresponding crack opening at peak bridging stress, $w_{B, 1}$. When multiple bridging stress peaks develop, distinct tensile hardening-softening sequences can be distinguished. In each of these tensile hardening-softening sequences, the local initial minimum and peak bridging stresses are distinguished by using the lower-case $b$ or the capital $B$ as subscripts, respectively. The tensile hardening-softening sequence $k$ will have, as main parameters, the local initial bridging stress $\sigma_{b, k}$, the local peak bridging stress $\sigma_{B, k}$ and the corresponding crack openings $w_{b, k}$ and $w_{B, k}$. The index $k$ can adopt the integer value from 2 to $n$, being $n$ the total number of tensile hardening-softening sequences. Finally, the residual bridging stress, $\sigma_{b, n+1}$, the corresponding crack opening, $w_{b, n+1}$, and the ultimate cohesive crack opening, $w_{u}$, characterize the last portion of the tensile bridging stress-crack opening curve, where the exhaustion of the remaining links between the two opposite crack surfaces 


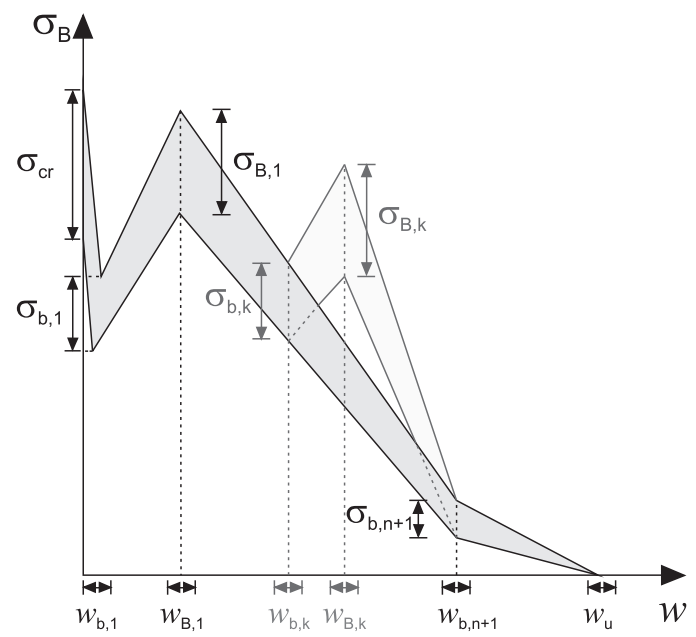

Figure 4: Schematic of tensile stress-crack opening response of cementitious composites reinforced with multiple fiber types.

occurs. The scatter of results associated with each parameter is also relevant in the modeling and up-scaling of the mechanical parameters derived with the SCTT to the structural level, as represented in Figure 4 by the shaded area.

The experimental results obtained for a few of the aforementioned parameters are presented in Table 4 . The compressive strength, $\sigma_{f c}$, and the first cracking stress, $\sigma_{c r}$, were selected due to their relation to the mechanical properties of the cementitious matrix. Conversely, the peak bridging stress, $\sigma_{B, 1}$, and the ultimate cohesive crack opening, $w_{u}$, were selected due to their sensitivity to the type of fiber reinforcement used. For practical reasons, in the present case $w_{u}$ was assumed as the crack opening observed for a residual tensile strength of 0.5 MPa. The average (avg), the standard deviation (std) and the coefficient of variation $(c v)$ were computed for each parameter in Table 4. 
251

Table 4: Main parameters and scatter obtained from compression and tension tests.

\begin{tabular}{|c|c|c|c|c|c|}
\hline \multirow{2}{*}{ Composite } & & \multirow{2}{*}{$\begin{array}{c}\sigma_{f c} \\
\mathrm{MPa} \\
(\%)\end{array}$} & \multirow{2}{*}{$\begin{array}{c}\sigma_{c r} \\
\mathrm{MPa} \\
(\%)\end{array}$} & \multirow{2}{*}{$\begin{array}{c}\sigma_{B, 1} \\
\mathrm{MPa} \\
(\%)\end{array}$} & \multirow{2}{*}{$\begin{array}{c}w_{u} \\
\mathrm{~mm} \\
(\%)\end{array}$} \\
\hline & & & & & \\
\hline \multirow{3}{*}{ 1PVA } & $a v g$ & 68.17 & 3.98 & 3.77 & 0.87 \\
\hline & std & 5.16 & 0.70 & 0.25 & 0.12 \\
\hline & $c v$ & $(7.6)$ & $(17.2)$ & $(6.6)$ & (13.4) \\
\hline \multirow{3}{*}{$2 \mathrm{PVA}$} & $a v g$ & 70.51 & 4.36 & 6.64 & 1.20 \\
\hline & std & 6.56 & 0.44 & 0.43 & 0.13 \\
\hline & $c v$ & $(9.3)$ & $(10.0)$ & $(6.4)$ & $(11.0)$ \\
\hline \multirow{3}{*}{ HybPAN } & $a v g$ & 89.53 & 5.25 & 4.64 & 0.64 \\
\hline & std & 5.60 & 0.84 & 0.48 & 0.07 \\
\hline & $c v$ & $(6.3)$ & $(16.0)$ & $(10.2)$ & (11.4) \\
\hline \multirow{3}{*}{$2 \mathrm{PAN}$} & $a v g$ & 54.02 & 4.99 & 5.03 & 0.15 \\
\hline & std & 6.17 & 0.77 & 0.42 & 0.01 \\
\hline & $c v$ & (11.4) & $(15.5)$ & $(8.4)$ & $(5.9)$ \\
\hline \multirow{3}{*}{ HybPP } & $a v g$ & 52.37 & 2.95 & 4.05 & $>2.0$ \\
\hline & std & 2.80 & 0.69 & 0.54 & - \\
\hline & $c v$ & $(5.3)$ & $(23.2)$ & (13.4) & - \\
\hline \multirow{3}{*}{$2.5 \mathrm{PP}$} & $a v g$ & 70.48 & 2.99 & 4.33 & $>2.0$ \\
\hline & std & 3.12 & 0.96 & 0.35 & - \\
\hline & $c v$ & $(4.4)$ & $(32.2)$ & $(8.1)$ & - \\
\hline
\end{tabular}


As shown in Table 4, the scatter of the experimental results was relatively low. The $c v$ derived for all parameters was below 15\%, with the exception of $\sigma_{c r}$. For both the composites reinforced with PP fibers the value of $w_{u}$ was not determined, since the test sequence was terminated at a CMOD of 2.0 $\mathrm{mm}$ for all specimens. In the case of the PP fiber reinforced composites, the tensile stresses measured at this CMOD were still higher than 0.5 MPa.

In Figure 5 the experimental tensile stress-crack opening curves are presented. For clarity, the experimental average curves were obtained by averaging the tensile stresses measured at each crack opening observed in all the tested specimens of every composite. Additionally, the envelope of all experimental results is represented by the shaded area.

\section{Discussion of results}

The results presented in Table 4 summarize the essential mechanical parameters characterizing the tensile response of the fiber reinforced cementitious composites tested. The robustness of the results is supported by the overall low statistical scatter obtained, with the exception of the first cracking stress results.

Although all composites had the same matrix composition, the compressive strengths obtained were clearly different. The average compressive strength results ranged between a minimum of $52 \mathrm{MPa}$ and a maximum of $90 \mathrm{MPa}$. The maximum $c v$ was $11.4 \%$, suggesting that the computed average compressive strengths are representative and satisfactorily robust. The clearly different results obtained show that the compressive strength of the 

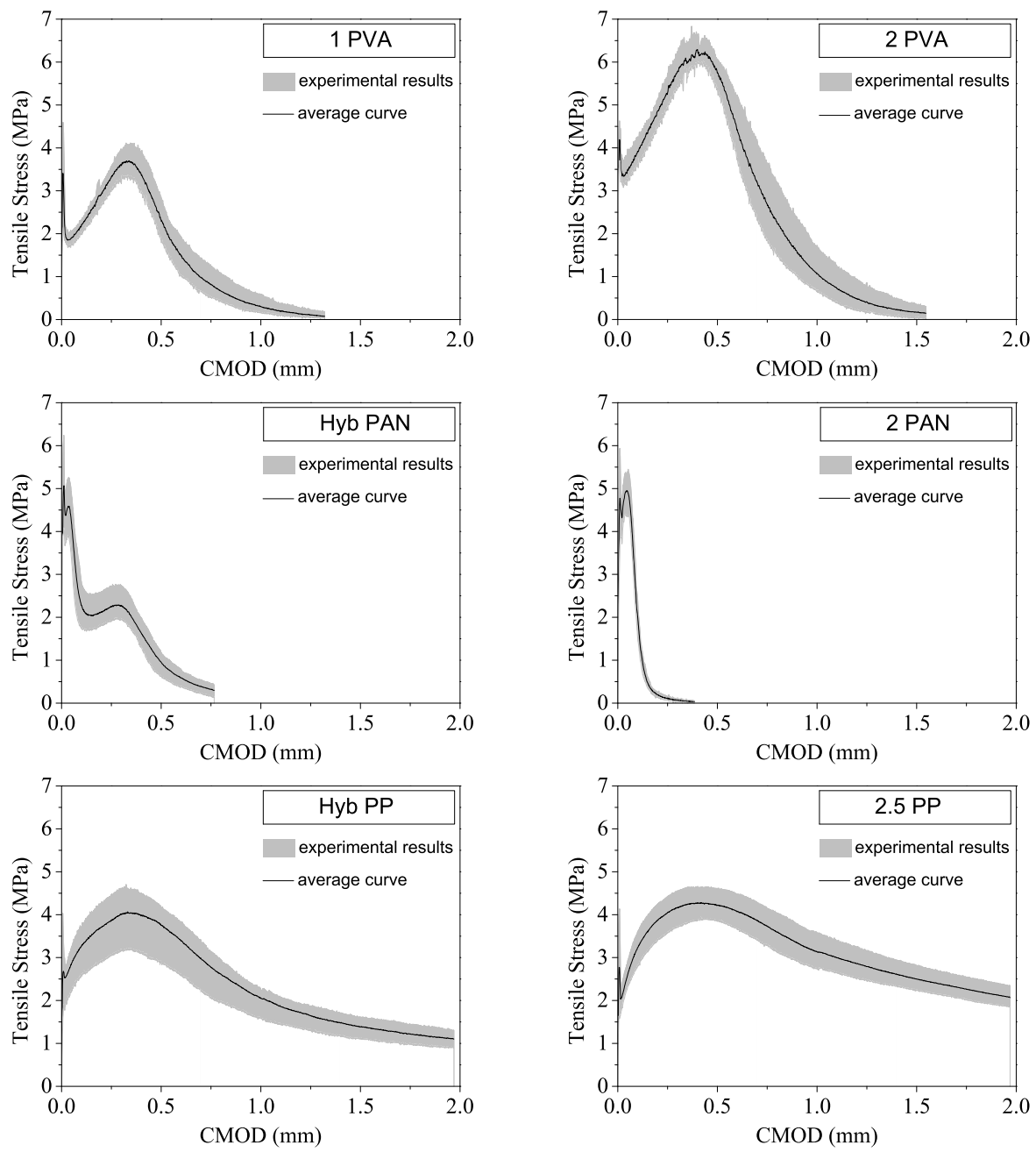

Figure 5: Average curves and envelopes of the experimental results obtained from six specimens of each composite.

composite is not only determined by the composition of the matrix. Consequently, either in a direct or indirect fashion, the fibers have an important influence in the compressive behavior of the composite. When designing the mix composition for a specific compressive strength class, the consideration of the contribution of different fiber types and volumes is therefore relevant. 
The results of the average first cracking stresses also showed a clear influence of the fiber reinforcement type. Either due to the direct contribution of fibers to restrain crack propagation or due to their interference with the micro-structure of the solid skeleton and the composite flaw size and distribution, these results show that the first cracking stress is not exclusively influenced by the matrix composition. The $c v$ values derived were noticeably higher, probably due to the greater influence of brittle fracture mechanisms and the typically associated scatter. While the $c v$ clearly differs when comparing the different composites tested, the $s t d$ is, however, of the same order of magnitude, suggesting that the mechanism causing this scatter is common to all tested composites. The present research is not sufficiently detailed to clarify this mechanism, but probably the results are strongly affected by the size distribution and type of porosity in the matrix, or by the effect of the aggregates used on the initiation of cracking, or any other factor with significant influence on the fracture processes and crack propagation in the composites.

The peak bridging stress may be considered as the mechanical parameter that is most strongly correlated with the characteristics of the fiber reinforcement adopted in each composite. The lower $c v$ values of the peak bridging stresses may be justified by the greater influence of the properties of the fiber reinforcement. In general these have low scatter, as a result of quality control in the production of the fibers.

When comparing the experimental results of all composites, no clear correlation between the average peak bridging stresses and the average compressive strengths is perceptible. Given that the matrix composition is identical 
in all tested composites, each type of fiber reinforcement seems to influence differently the compressive and the tensile properties of each composite. Similarly, no correlation is perceptible between the first cracking stress and the compressive strength or the peak bridging stress. These results may be explained at the level of the composite micro-mechanics, of great complexity. Their complete understanding may be circumvented if a robust procedure is employed to describe the fundamental mechanical parameters of the tensile composite behavior, both in the material design and in the constitutive modeling viewpoints. This procedure should be sensitive to the main composite parameters, like the nature and geometry of fiber reinforcements used, the matrix mechanical properties and the interaction that is established between them.

Using the SCTT setup previously described, in this research six distinctive tensile stress-crack opening behaviors were identified. The experimental results shown in Figure 5 demonstrate that the tensile stress-crack opening curve is very sensitive to the characteristics of the fiber reinforcements used. As shown in Figure 5, the effective contribution of the fibers to the tensile-hardening mechanisms occurred at clearly different CMOD of the tensile stress-crack opening behavior. The different orders of magnitude of the CMOD at which the diverse fibers showed greater efficiency may be one motivation to support the typically idealized multi-scale nature of the mechanics of cementitious composites in tension, when multiple types of fibers are employed. In general, the composites showed singular tensile bridging stress hardening-softening sequences $(n=1$, see Figure 4$)$. The exception was the composite containing 1\%PVA $+1 \%$ PAN fibers (HybPAN), where two dis- 
tinct tensile hardening-softening sequences were identified ( $n=2$, see Figure 4). Hence, the tensile response in terms of the tensile stress-crack opening behavior clearly revealed the use of two distinct types of fibers in the composite.

The experimental results show that the mechanical contribution of the smaller and thinner fibers was exhausted at a much earlier stage, that is, at relatively small crack widths. The tensile capacity of the composite reinforced with $2 \%$ of PAN fibers was fully exhausted before most of the other composites reached their respective peak bridging stresses. In contrast, the longer PP fibers provided significant contribution to the tensile strength of the composites for almost the entire range of CMOD measured, reaching the peak bridging stress at larger CMOD and showing higher residual tensile strengths. The PVA fibers developed the highest bridging stresses in the composite and contributed more effectively to the composite tensile strength at intermediate CMOD values.

\section{Micro-mechanics of cracking at multiple scales of the cracking process}

The results presented in Figure 5 show that the effective mobilization of the different types of fibers during the SCTT sequence occurs at different CMOD ranges, which correspond to different length scales of the material micro-structure. The PAN fibers restrain crack opening at small crack widths, reaching the peak bridging stress at a CMOD of approximately 50 $\mu \mathrm{m}$. In contrast, the composites reinforced with PP fibers show the little con- 
tribution of the fibers to crack restraint at smaller CMOD. Instead, PP fibers are more effective at maintaining high levels of residual tensile strengths up to the maximum CMOD adopted in the experimental program (2 mm). The peak bridging stress in the PP fiber reinforced composite is reached at a CMOD of approximately $500 \mu \mathrm{m}$, one order of magnitude above the CMOD at peak bridging stress observed in the PAN fiber reinforced composite. The scale of the cracking process at which PAN and PP fibers are effective is, therefore, clearly different.

\subsection{Tensile response up to $50 \mu \mathrm{m}$}

The discussion of the micro-mechanisms underlying the observed tensile behaviors requires a closer observation of each distinct cracking stage at different scales of the cracking process. In Figure 6, the tensile behavior observed for all composites is presented up to a CMOD of $50 \mu \mathrm{m}$. The average curves presented earlier for the composites reinforced with PAN and PP fibers are shown in addition to the average curves obtained for the composites reinforced with $1 \%$ and $2 \%$ PVA fibers.

The tensile stress-crack opening curves presented in Figure 6 show that the contributions provided by the different fiber reinforcements up to a CMOD of $50 \mu \mathrm{m}$ are clearly differentiated. The tensile responses diverge from the linear elastic behavior almost right from the onset of the testing sequence. The high stress concentrations created at the tip of the notch in the specimens lead to the early initiation and propagation of the crack plane. Therefore, some fibers crossing the notched cross-section plane near the tip 


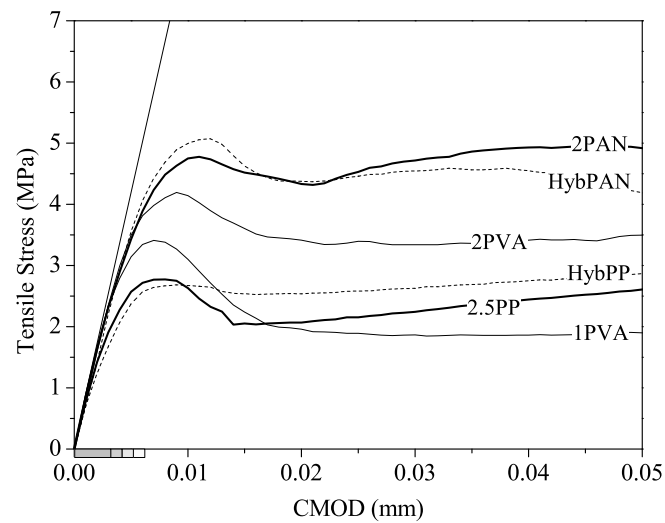

Figure 6: Tensile responses up to a CMOD of $50 \mu \mathrm{m}$

become active before the first cracking stress $\left(\sigma_{c r}\right.$, see Figure 4$)$ is reached. In general all types of fibers contribute to the first cracking stress, although this contribution may be more or less significant, depending of the nature and geometry of the fiber. At this smaller scale of observation, $\sigma_{c r}$ represents the the peak stress reached up to a CMOD of $10 \mu \mathrm{m}$. The early crack initiation obtained in a controlled manner with the SCTT setup allows distinguishing the different contributions provided by different fibers at very small crack widths. For the same matrix, PAN fibers showed a more effective contribution to crack restraint at this stage, followed by the PVA fibers and by the PP fibers. The average value of $\sigma_{c r}$ ranges from $2.95 \mathrm{MPa}$ for the composites containing PP fibers, to 5.25 MPa for the composites containing PAN fibers. The load decay subsequent to cracking is apparently smoother in the case of the composite reinforced with the PAN fibers. Although the transfer of the tensile stresses from the matrix to the fibers occurs at a higher tensile load, the effective mobilization of PAN fibers at this stage is contributing to a smoother load decay. In contrast, even at lower tensile stresses, the post- 
peak load decay observed with the PP fiber reinforced composites occurs rapidly, probably due to the lower interfacial bonding of these fibers with the matrix and the consequent earlier fiber debonding. The remaining part of the observed length scale in Figure 6 is also dominated by the PAN fibers, which reveal a superior contribution to crack restraining at CMOD up to 50 $\mu \mathrm{m}$.

\subsection{Tensile response up to $0.5 \mathrm{~mm}$}

In Figure 7 the tensile behavior obtained for all composites is presented for a CMOD up to $0.50 \mathrm{~mm}$. At this length scale of the cracking process, the PVA fibers clearly show the more effective contribution to crack restraining. The composites reinforced with $2 \%$ PVA reached the highest peak bridging stress at a CMOD of approximately $0.40 \mathrm{~mm}$. The PP fibers also contributed considerably to the tensile bridging stresses observed at this scale, but the lower interfacial bond between the PP fiber surface and the matrix led to the gradual loss of crack bridging capacity at increasing CMOD. These lower interfacial bond properties also led to lower initial bridging stresses $\left(\sigma_{b, 1}\right.$, see Figure 4) and to a more abrupt load decay at aprevious stage. This effect was attenuated when a fraction of the PP fibers was replaced by PVA fibers, in the case of the hybrid composite $(1 \% \mathrm{PVA}+1.25 \% \mathrm{PP})$.

In general the results show that, when using PAN fibers, the small fiber diameter and the improved bonding with the matrix enhance the crack restraint for CMOD smaller than $10 \mu \mathrm{m}$, consequently the $\sigma_{c r}$ reached is higher. The subsequent load decay, followed by a tensile hardening stage up to an 


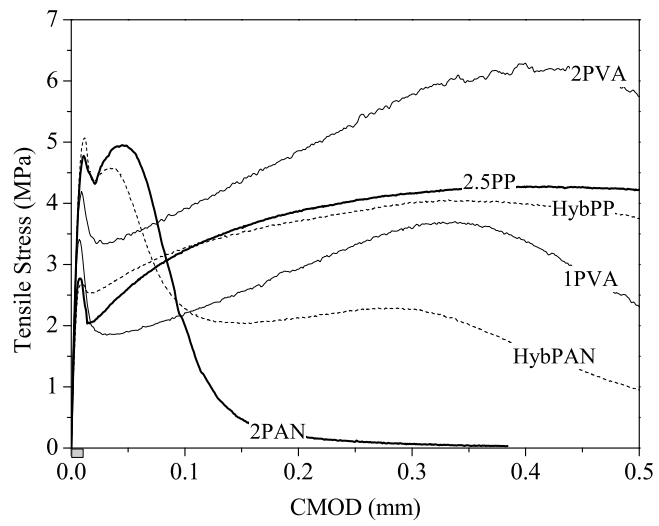

Figure 7: Tensile responses up to a CMOD of $0.50 \mathrm{~mm}$

approximate CMOD of $50 \mu \mathrm{m}$, are the consequence of the gradual mobilization of the PAN fibers present in the composite, which remain as the most effective at the smaller length scale of the cracking process. After the peak bridging stress is reached, the subsequent stress decay is rapid, as shown in Figure 7. When half of the PAN fiber reinforcement is replaced by PVA, some of the crack restraining effect at the early cracking stage is lost. However, the $1 \% \mathrm{PVA}+1 \% \mathrm{PAN}$ composite developed a second tensile hardening sequence $(n=2$, see Figure 4$)$ at higher CMOD values. While aggregating the features observed in each of the single fiber composites, the hybrid seems to underperform the single fiber composites at the cracking stages where the latter individually showed higher effectiveness. In the case of the PVA+PAN composite, this effect was less significant at smaller CMOD (up to $50 \mu \mathrm{m}$ ). At higher CMOD, the hybrid underperformed even the composite containing $1 \%$ of PVA. The loss of workability of the fresh mixtures due to the addition of PAN fibers may justify this result. 


\subsection{Tensile response up to $2.0 \mathrm{~mm}$}

The use of the PP fibers resulted in a delayed mobilization of the fiber reinforcement. As shown in Figure 8, the tensile stress-crack opening response of the composite reinforced with $2.5 \%$ PP fibers presented a long tail of relatively high tensile bridging stresses in the entire range of measured CMOD. Due to the reduced interfacial bond of the fibers with the surrounding matrix, the prevailing pull-out mechanism of PP fibers manifests itself both in the early stages of cracking (Figure 6) and in the overall behavior (Figure 8). During the early stage of cracking, the contribution of the PP fibers to the first cracking stress was less significant than the one observed for the PVA fiber reinforced composites. After cracking, the load decay observed in the PP fiber reinforced composites was more significant and the first bridging stress $\left(\sigma_{b, 1}\right.$, see Figure 4$)$ was lower. This is the consequence of the rapid propagation of the tunnel crack during debonding, with the transfer of the tensile stresses form the matrix to the fibers. The subsequent fiber bridging stress recovery was also steeper in the $\mathrm{PP}$ reinforced composites, as a consequence of the low stress $\left(\sigma_{b, 1}\right)$ at which the high fiber reinforcement ratio is being mobilized. With the increase of the CMOD, the initial stiffness observed in the tensile hardening stage gradually decreases, as result of the prevailing fiber pull-out mechanisms.

The main difference between the SCTT results observed in PVA and PP fiber reinforced composites is located at the region where the peak bridging stress is reached $\left(\sigma_{B, 1}\right.$, see Figure 4$)$. While in the PVA fiber reinforced composites the experimental curves exhibited a sharp transition from the 


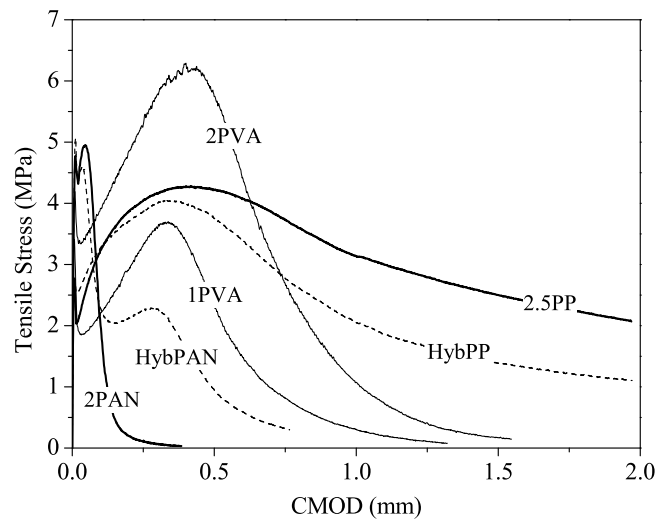

Figure 8: Tensile responses up to a CMOD of $2.0 \mathrm{~mm}$

tensile hardening to the tensile softening stages, in the PP fiber reinforced composites the same transition is smooth and gradual (Figure 8). The well known superior interfacial bonding of the PVA fibers with the matrix may justify these results, as opposed to the lower bonding of the PP fibers due to their hydrophobic nature $[31,32]$. In the PP fiber reinforced composites the gradual increase of the stress transferred to the fibers during the post-cracking tensile hardening stage results in the early debonding of a gradually increasing number of PP fibers. Therefore, the initial tensile hardening stiffness steadily decreases at increasing CMOD and the transition to the softening phase occurs gradually, generating the typical smoothly curved shape of the tensile response in the vicinity of the peak bridging stress. The superior bonding of the PVA fibers with the matrix results in a constant tensile hardening stiffness during most of the post-cracking tensile hardening stage. This improved bond behavior is also responsible for the rupture of most of the fibers once the tensile load approaches the bridging peak. Therefore, after the peak bridging stress is reached, tensile softening occurs more 
rapidly in the PVA than in the PP fiber reinforced composites. Additionally, this differentiated bond behavior also explains the preservation of a higher residual tensile strength in the composites reinforced with PP fibers. The hybrid composite $(1 \% \mathrm{PVA}+1.25 \% \mathrm{PP})$ showed a mixed behavior, aggregating the features observed in the corresponding composites reinforced with fibers of single type. As before, the hybrid composite underperformed the respective composites reinforced with single types of fibers at the cracking stages where the latter, individually, showed a more effective tensile response.

\subsection{Energy dissipated in the fracture process}

An important indicator of the overall tensile performance of the tested composites is the energy dissipated during the fracture process. For the better understanding of the role of each type of fiber in each of the cracking process length scales previously referred, the energy dissipated during the fracture process was computed at three distinct CMOD: $50 \mu \mathrm{m}, 0.50 \mathrm{~mm}$ and $2.0 \mathrm{~mm}$. In Figure 9, the results are presented for the six composites tested. These results were based on the average experimental tensile stresscrack opening curves of each composite.

In general, the results show that the most effective type of fiber is not the same at each of the represented length scales of the cracking process. Up to a CMOD of $50 \mu \mathrm{m}$, both in the single fiber and in the hybrid fiber reinforced composites, the PAN fibers exhibit higher effectiveness. The PVA fibers are more effective in the intermediate scale, showing the highest dissipated energies at a CMOD of $0.50 \mathrm{~mm}$. PP fibers have significant effect at larger crack openings, showing both the composites containing PP fibers the 


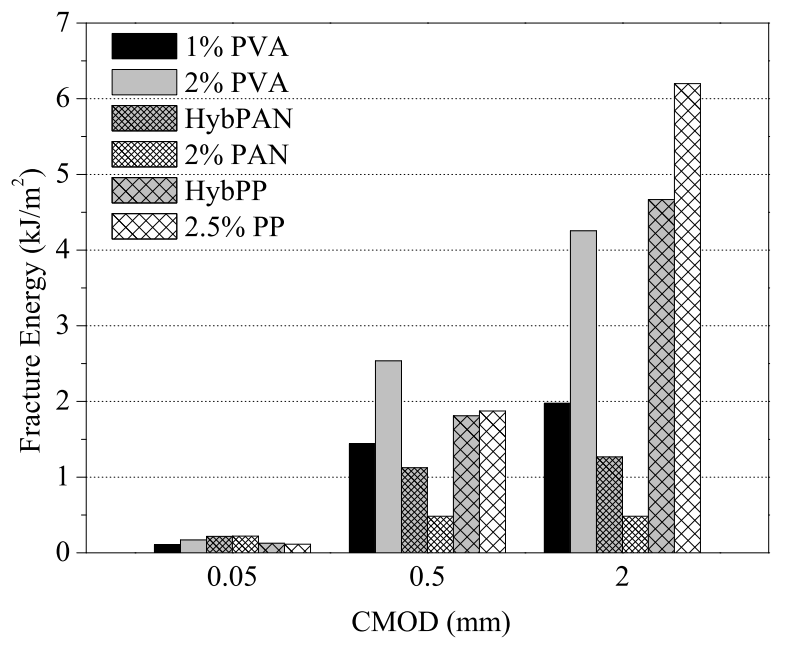

Figure 9: Energy dissipated during fracture computed for a maximum CMOD of $50 \mu \mathrm{m}$, $0.50 \mathrm{~mm}$ and $2.0 \mathrm{~mm}$, based on the average tensile stress-crack opening curves.

highest energy dissipation at a CMOD of $2.0 \mathrm{~mm}$. Considering the potential synergistic effect of having multiple fiber-type reinforcements, the hybrid composites were expected to outperform the superposition of the corresponding single fiber reinforced composites. A positive synergistic effect cannot be observed. In the special case of the hybrid composite reinforced with PVA and PAN fibers, the hybrid even underperformed the 1\% PVA reinforced composite at CMOD between 0.1 and $0.5 \mathrm{~mm}$. One possible explanation for this result is the unfavorable effect of PAN fibers on the fresh properties of the composite. 


\section{Effectiveness of hybrid fiber reinforcement}

In order to clarify the effectiveness of the hybrid fiber reinforcements, the tensile response of the hybrid composites was predicted based on the results obtained from the corresponding single fiber reinforced composites. To this purpose, the expected tensile stress-crack opening curves of the hybrid fiber reinforced composites were obtained by superposing the contribution of each fiber fraction. The hybrid fiber reinforced composites contain $50 \%$ of the amount of fiber reinforcement used in the corresponding single fiber reinforced composites, therefore the contribution of each type of fiber in the hybrid composites was assumed as equal to $50 \%$ of the tensile stress measured at each CMOD of the corresponding single fiber reinforced composites. The total volume of fiber reinforcement in the single fiber and in the hybrid fiber reinforced composites is, thus, nearly identical to the volume of fiber reinforcement assumed in the expected tensile responses. This is essential to the assumption of a comparable micro-structural arrangement of the solid particles and dispersed fibers in the composites, for both the experimental and the expected tensile responses.

\subsection{Hybrid fiber reinforcement of $P V A$ and $P A N$ fibers}

In Figure 10, the composite HybPAN (1\%PVA + 1\%PAN) expected tensile stress-crack opening response is compared to the experimental. Additionally, the experimental responses of the corresponding single fiber reinforced composites (2\% PVA and $2 \% \mathrm{PAN})$ are shown. The gray shaded area represents the envelope of the expectable tensile responses to the hybrid fiber 
reinforced composite, when assuming that this tensile response is the synthesis of the tensile responses obtained for the corresponding single fiber reinforced composites. Exemplifying, one boundary of this gray shaded area is obtained when the expected hybrid fiber composite tensile response is the result of adopting a ratio of $100 \%$ of the $2 \%$ PVA tensile response and $0 \%$ of the $2 \%$ PAN tensile response, which coincides with the experimental tensile response obtained for the $2 \%$ PVA fiber reinforced composite. The other boundary of this gray shaded area is obtained when the ratio $0 \%$ of the $2 \% \mathrm{PVA}$ tensile response and $100 \%$ of the $2 \% \mathrm{PAN}$ tensile response are considered, and the expected tensile response for the hybrid coincides with the $2 \%$ PAN fiber reinforced composite. The tensile response obtained when $50 \%$ of the $2 \%$ PVA tensile response and $50 \%$ of the $2 \%$ PAN tensile response are considered corresponds to the expected tensile response for the $1 \% \mathrm{PVA}$ $+1 \%$ PAN hybrid fiber reinforced composite. This expected tensile response can thus be compared to the experimentally obtained tensile response for the $1 \% \mathrm{PVA}+1 \% \mathrm{PAN}$ hybrid fiber reinforced composite, with the purpose of investigating possible synergistic effects emerging from the simultaneous use of two different types of fibers in the same cementitious composite.

The experimental tensile bridging stresses of the hybrid fiber reinforced composite never exceed the upper limit of the gray area, as shown Figure 10. Therefore, the hybrid fiber reinforced composite is unable to perform better than the corresponding single fiber reinforced composites in the regions of the tensile response where the latter are more effective. Comparing the expected and the experimental tensile responses obtained for the hybrid fiber 


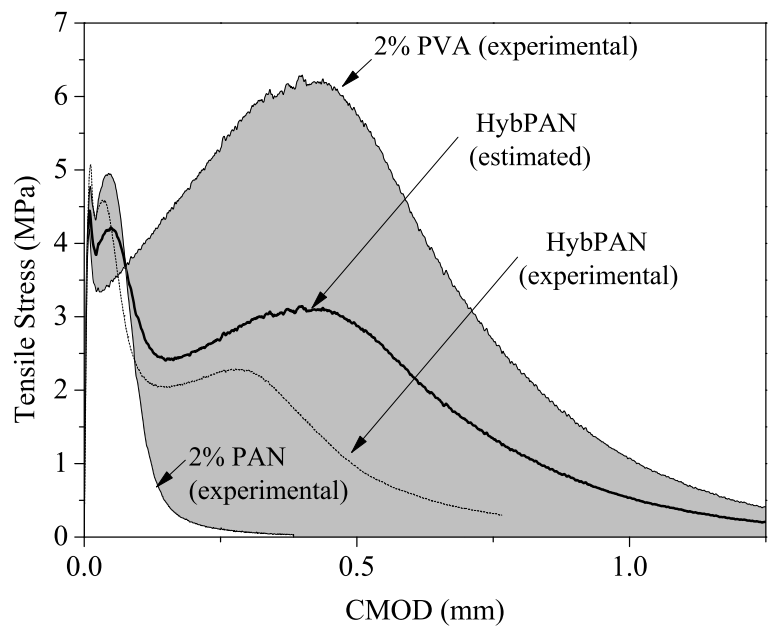

Figure 10: Expected tensile response of the hybrid fiber reinforced composite (PVA, PAN) based on the tensile responses of the corresponding single fiber reinforced composites.

reinforced composite, only at very small CMOD the experimental curve outperforms the expected one. Only at this small region of the tensile response a probable positive synergy may be hypothesized. Therefore, the presence of different types of fibers in the same composite does not necessarily lead to better tensile performance. In fact, the achievement of a better performance when compared with the one resulting from the superposition of the contributions of each fiber fraction is unlikely, since the regions of the tensile response where the optimal mobilization of each type of fiber occurs are different. As a consequence, the activation of the fibers during the fracture process is not synchronized, which causes a reduction of the overall tensile performance. In addition, fiber reinforcement mechanisms of individual fibers are not optimized when several types of fibers are used in the same composite, since it is unlikely that the optimal matrix properties considering the interaction of 
one type of fiber with the matrix, are simultaneously optimal to the other types of fibers.

The multi-scale nature of the cracking process, and the adoption of different fiber reinforcement types in crack restraining at the level of these different length scales of the cracking process, are generally recognized as fundamental principles leading to optimized fiber reinforcement design concerning tensile performance. The length and diameter of the PVA and PAN fibers used in this study were clearly different, and the experimental results showed that the crack restraining effect provided by these fibers was occurring at clearly distinct length scales of the cracking process. However, a positive synergy between these two different fibers in the hybrid composite was not observed. Only at very small CMOD the experimental curve surpassed the expected one, suggesting that in the early stage of crack formation a synergistic collaboration of the fibers to crack restraining may have occurred. This may, however, not be sufficient to support the general use of hybrid fiber formulations, if optimal tensile performance is pursued. Nevertheless, the doublepeak nature of the tensile stress-crack opening response was a unique feature demonstrated by the HybPAN composite, which may be of interest for particular applications.

\subsection{Hybrid fiber reinforcement of $P V A$ and PP fibers}

In Figure 11 the HybPP $(1 \% \mathrm{PVA}+1.25 \% \mathrm{PP})$ expected tensile stresscrack opening curve is compared to the tensile response experimentally obtained. The tensile responses experimentally obtained for the corresponding single fiber reinforced composites $(2 \% \mathrm{PVA}$ and $2.5 \% \mathrm{PP})$ are also shown. As 
in the previous section, the envelope of the gray shaded area is established by the tensile responses experimentally obtained for the composites reinforced with $2 \% \mathrm{PVA}$ and with $2.5 \% \mathrm{PP}$, which are the hypothetical limits of the hybrid fiber reinforced composite expected tensile response.

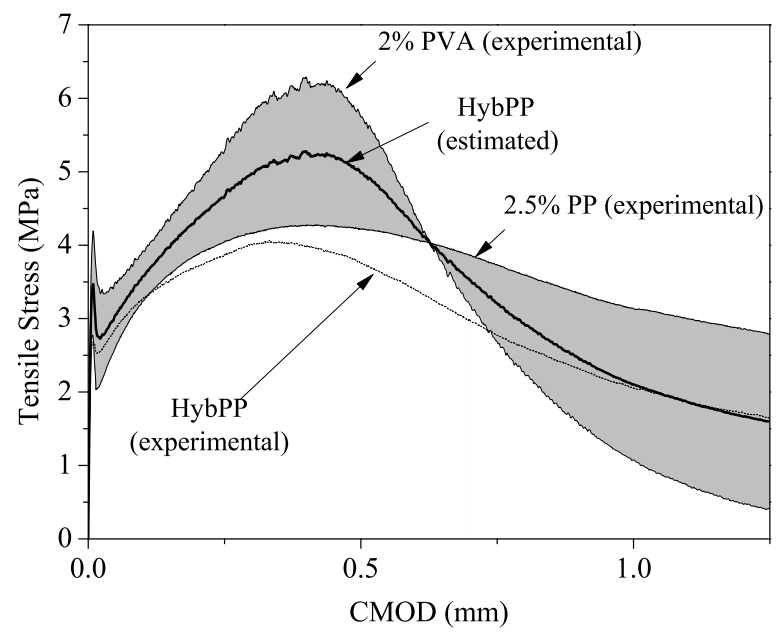

Figure 11: Expected tensile response of the hybrid fiber reinforced composite (PVA, PP) based on the tensile responses of the corresponding single fiber reinforced composites.

As shown in Figure 11, the tensile response of the hybrid composite never surpasses the upper limit of the gray area. Therefore, also when using PVA and PP fibers as reinforcement, the hybrid composite is unable to perform better than the corresponding single fiber reinforced composites in the regions of the tensile response where the latter are more effective. Comparing the expected and the experimental tensile responses obtained for the HybPP $(1 \% \mathrm{PVA}+1.25 \% \mathrm{PP})$, the experimental response also underperforms the expected response in the entire range of CMOD measured. The discrepancy 
between the expected and the experimental responses is higher in the vicinity of the peak bridging stress, that is, in the region of the tensile response where the optimal mobilization of the fibers is supposed to occur. Although the tensile responses obtained from the single fiber reinforced composites (2\% PVA and $2.5 \% \mathrm{PP})$ are substantially different, the region of the tensile response where optimal mobilization of the fibers occurs is essentially identical, and the CMOD at which the peak bridging stress is reached is nearly identical. This may suggest that a synchronized mobilization of the fibers is occurring in the hybrid composite, but the experimentally obtained tensile response for the hybrid fiber reinforced composite clearly underperforms the expected tensile response. Therefore the activation of the different types of fibers may not be fully synchronized, in this case not because fiber full mobilization occurs at different CMOD, but because the micro-mechanisms of fiber mobilization are essentially distinct for PVA and for PP fibers. In particular, as a result of the lower interfacial bond between the PP fibers and the matrix, the fiber length required to transfer the tensile stresses from the matrix to the fibers is longer in PP than in the PVA fibers. The different mechanics of interaction between the matrix and the PVA or the PP fibers result in considerable differences in the micro-mechanisms of crack restraining provided by the PVA or the PP fibers. The result is the formation of substantially different stress fields in the transition zone between the fiber bridged crack plane and the intact bulk material, depending on the type of fiber considered. This discrepancy may justify the loss of performance observed in the hybrid fiber reinforced composite, and may be interpreted as a lack of synchronism of the crack restraining micro-mechanisms between PVA 
and PP fibers. Additionally, the different nature of the fiber-matrix interaction for the different fibers in the fresh mixture probably led to poorer fiber dispersion during mixing, with negative consequences to the tensile performance of the hardened composite.

\section{Conclusions}

In this research, the single crack tension test (SCTT) was used to assess the tensile performance of six fiber reinforced cementitious composites. The responses obtained showed high sensitivity to the parameters of the different cementitious composites tested, in particular to the different types of fiber reinforcements used. The experimental results showed considerable robustness and reasonably low dispersion of results. This reduced dispersion contributed to the clarification and effective understanding of the fiber-matrix interactions and of the the different stages of crack initiation and propagation.

The experimental tensile stress-crack opening responses showed that the different fibers used in the composites are activated at different length scales of the cracking process. The small diameter fibers (PAN) showed a more effective contribution to crack restraining at very small crack openings, almost ten times smaller than the observed for the larger diameter fibers (PVA, PP). This differentiated contribution of the fibers to crack restraining at different scales of the cracking process was also revealed by the multiple tensile hardening-softening sequences observed in one of the hybrid composites. The generic shape of a multiple-peak tensile stress-crack opening relationship was suggested, considering the most important micro-cracking mechanisms and 
the main stages of the cracking processes.

The multi-scale nature of cracking mechanisms in cementitious matrix composites is often assumed as the reason to support the adoption of hybrid fiber reinforcements. Although the experimental results have shown that these different length scales of the cracking process exist, and that the activation of different fibers occurs at different scales of the material structure, the synergistic effect of combining different types of fibers in the same composite could not observed. The hybrid formulations underperformed the respective single fiber-type reinforced composites at the CMOD ranges where the latter were more effective. In addition, the hybrid formulations also underperformed the expected tensile performances based on the direct superposition of the tensile responses of the corresponding single fiber-type reinforced composites. The multiple peaks of the tensile bridging stress reached in the composite containing two geometrically distinct types of fibers may be of interest for further investigation, for special applications. However, considering the optimization of the composite tensile performance, a design process leading to the synchronized activation of the fibers intersecting a crack in the hybrid composite should be preferred.

The design of a hybrid fiber reinforced cementitious composite will eventually consist of selecting the length scale or scales of the cracking process at which the fiber crack restraining effect is more important. A multiplicity of different applications may present diverse requirements concerning the tensile stress-crack opening response of the cementitious composite, which for various reasons may justify the adoption of fiber reinforcements composed of two or more different types of fibers. However, the design of optimal com- 
positions of fiber reinforcement towards optimized tensile performance must consider the need to preserve synchronism in the activation of the different types of fibers, which is not likely when multiple types of fibers are used simultaneously in the same cementitious composite. The results obtained suggest that, when a single fiber type is utilized and all fibers in the composite are similar, the synchronized activation of all fibers is more likely to occur and their contribution to crack restraining tends to be more effective.

\section{Acknowledgments}

The authors thank the Portuguese National Science Foundation for the financial support, through grant SFRH / BD / 36515 / 2007, funded by POPH-QREN, the Social European Fund and the MCTES, and DTU-Byg for their support of the work as part of this project.

\section{References}

[1] V. C. Li, Large volume, high-performance applications of fibers in civil engineering, Journal of Applied Polymer Science 83 (2002) 660-686.

[2] V. C. Li, On engineered cementitious composites (ecc), J Adv Conc Tech 1 (2003) 215-230.

[3] A. M. Brandt, Fibre reinforced cement-based (frc) composites after over 40 years of development in building and civil engineering, Compos Struct 86 (2008) 3-9. 
[4] S. F. U. Ahmed, M. Maalej, P. Paramasivam, Flexural responses of hybrid steel-polyethylene fiber reinforced cement composites containing high volume fly ash, Construction \& building materials 21 (2007) 10881097.

[5] N. Banthia, R. Gupta, Hybrid fiber reinforced concrete (hyfrc): Fiber synergy in high strength matrices, Mater Struct Materials and structures 37 (2004) 707-716.

[6] C. Qian, P. Stroeven, Fracture properties of concrete reinforced with steel-polypropylene hybrid fibres, Cement \& concrete composites 22 (2000) 343-351.

[7] J. Lawler, T. Wilhelm, D. Zampini, S. Shah, Fracture processes of hybrid fiber-reinforced mortar, Mater Struct Materials and structures 36 (2003) 197-208.

[8] P. Rossi, High performance multimodal fiber reinforced cement composites (hpmfrcc): the lcpc experience, ACI Mater J 94 (1997) 478-483.

[9] B. Mobasher, C. Y. Li, Mechanical properties of hybrid cement-based composites, ACI Mater J 93 (1996) 284-292.

[10] J. van Mier, Fracture processes of concrete: assesment of material parameters for fracture models, New directions in civil engineering, CRC Press, 1997.

[11] L. Betterman, C. Ouyang, S. Shah, Fiber-matrix interaction in microfiber-reinforced mortar, Advanced Cement Based Materials 2 (1995) 53-61. 
[12] B. Cotterell, Y.-W. Mai, Fracture mechanics of cementitious materials, Blackie, London, 1996.

[13] B. Karihaloo, Fracture mechanics and structural concrete, Longman Scientific and Technical, Harlow, 1995.

[14] S. Shah, S. Swartz, C. Ouyang, Fracture mechanics of concrete - Applications of fracture mechanics to concrete, rock, and other quasi-brittle materials, Wiley, New York,N.Y., 1995.

[15] G. Chanvillard, Characterization of fibre reinforced concrete's performance after a flexular test - part 2: Identification of an intrinsic behaviour relationship in tension caracterisation des performances d'un beton renforce de fibres a partir d'un essai de flexion. partie 2: Identification d'une loi de comportement intrinseque en traction, Mater Struct Materials and structures 32 (1999) 601-605.

[16] J. Barros, V. Cunha, A. Ribeiro, J. Antunes, Post-cracking behaviour of steel fibre reinforced concrete, Materials and Structures 38 (2005) $47-56$.

[17] I. Lofgren, H. Stang, J. F. Olesen, The wst method, a fracture mechanics test method for frc, Mater Struct 41 (2008) 197-211.

[18] J. Zhang, C. K. Y. Leung, S. Xu, Evaluation of fracture parameters of concrete from bending test using inverse analysis approach, Mater Struct 43 (2010) 857-874.

[19] G. Chanvillard, Characterization of fibre reinforced concrete's performance after a flexural test - on subjectivity of toughness indices carac- 
terisation des performances d'un beton renforce de fibres a partir d'un essai de flexion. partie 1: De la subjectivite des indices de tenacite, Mater Struct Materials and structures 32 (1999) 418-426.

[20] J. Bolander, J.E., S. Saito, Discrete modeling of short-fiber reinforcement in cementitious composites, Advanced Cement Based Materials 6 (1997) $76-86$.

[21] V. Cunha, J. Barros, J. Sena-Cruz, A finite element model with discrete embedded elements for fibre reinforced composites, Computers and Structures 94-95 (2012) 22-33.

[22] H. Stang, V. C. Li, H. Krenchel, Design and structural applications of stress-crack width relations in fibre reinforced concrete, Materials and Structures 28 (1995) 210-219.

[23] P. Kabele, Multiscale framework for modeling of fracture in high performance fiber reinforced cementitious composites, Eng Frac Mech 74 (2007) 194-209.

[24] P. Kabele, Finite element fracture analysis of reinforced shcc members, in: G. van Zijl, W. Boshoff (Eds.), Advances in Cement-Based Materials, volume International Conference on Advanced Concrete Materials, Stellenbosch, South Africa, pp. 237-244.

[25] G. Fischer, H. Stang, L. Dick-Nielsen, Initiation and development of cracking in ecc materials: Experimental observations and modeling, in: G. P. Carpinteri GFA. (Ed.), High Performance Concrete, Brick- Ma- 
sonry and Environmental Aspects., volume 3, FraMCos, Taylor \& Francis, 2007, pp. 1517-1522.

[26] J. Yang, G. Fischer, Implications of the fiber bridging stress - crack opening relationship on properties of fiber reinforced cementitious composites in uniaxial tension, in: International Workshop on High Performance Fiber Reinforced Cementitious Composites in Structural Applications, volume RILEM Proceedings PRO 49, pp. - .

[27] E. B. Pereira, G. Fischer, J. A. Barros, M. Lepech, Crack formation and tensile stress-crack opening behavior of fiber reinforced cementitious composites (frcc), 7th International Conference on Fracture Mechanics of Concrete and Concrete Structures (FraMCoS 7) (2010) -.

[28] E. Pereira, G. Fischer, J. Barros, Direct assessment of tensile stress-crack opening behavior of strain hardening cementitious composites (shcc), Cement and Concrete Research 42 (2012) 834-846.

[29] E. N. B. Pereira, J. A. O. Barros, A. Cames, Steel fiber-reinforced selfcompacting concrete: Experimental research and numerical simulation, J Struct Eng 134 (2008) 1310-1321.

[30] F. de Larrard, Concrete mixture proportioning: a scientific approach, Modern concrete technology series, E \& FN Spon, 1999.

[31] V. C. Li, S. Wang, A. Ogawa, T. Saito, C. Wu, Interface tailoring for strain-hardening polyvinyl alcohol-engineered cementitious composite (pva-ecc), ACI Mat J 99 (2002) 463-472. 
${ }_{798}$ [32] Q. F. Wei, R. R. Mather, A. F. Fotheringham, R. D. Yang, Esem study 799 of wetting of untreated and plasma treated polypropylene fibers, J Ind $800 \quad$ Text $32(2002) 59-66$. 